\title{
Images
}

\section{Diffuse soft tissue emphysema after dental procedure}

\author{
Chia-Chun Liu, MD*; Ming-Yee Lin, MD*
}

A 22-year-old male presented to the emergency department due to progressive odynophagia and dysphagia. He underwent a left third mandibular molar extraction at the dental clinic a week prior. At the emergency department, vital signs were normal except tachycardia (114/min) and tachypnea (22/min). A physical examination revealed bilateral neck tenderness with crepitus on palpation, no stridor or acute airway compromise signs. Laboratory data showed leukocytosis (white blood cell count, 19000/cumm; segmented, $91 \%)$ and elevated C-reactive protein $(1.07 \mathrm{mg} / \mathrm{dL})$. Radiographs showed the presence of prevertebral emphysema on the C-spine lateral view (Figure 1) and subcutaneous emphysema with pneumomediastinum on the chest posteroanterior view (Figure 2). A noncontrast computed tomography (CT) scan confirmed the radiographic findings; additionally, there was gas collection over the pericarotid, pericardial (Figure 3) and extradural spaces (Figure 4). Because there was no CT evidence of infectious signs and clinical features were stable, conservative treatment with intravenous amoxicillin/clavulanic acid was given for a week. Clinical symptoms improved, and a follow-up CT showed no residual gas.

Although subcutaneous emphysema is a rare complication following dental procedures, with mostly benign and self-limiting sequelae, it may progress to fatal consequences such as cardiac tamponade, air embolism, pneumothorax, and mediastinitis. ${ }^{1}$ There have been reports correlating subcutaneous emphysema and dental treatments with the use of air turbine hand-pieces and air syringes. ${ }^{2,3} \mathrm{CT}$ is useful to detect the precise extension of gas dissection, such as pneumomediastinum, pneumopericardium, and pneumorrhachis, in this case. ${ }^{4}$ Most importantly, CT can evaluate the complications of extensive emphysema or infectious process. In addition, it can guide clinical

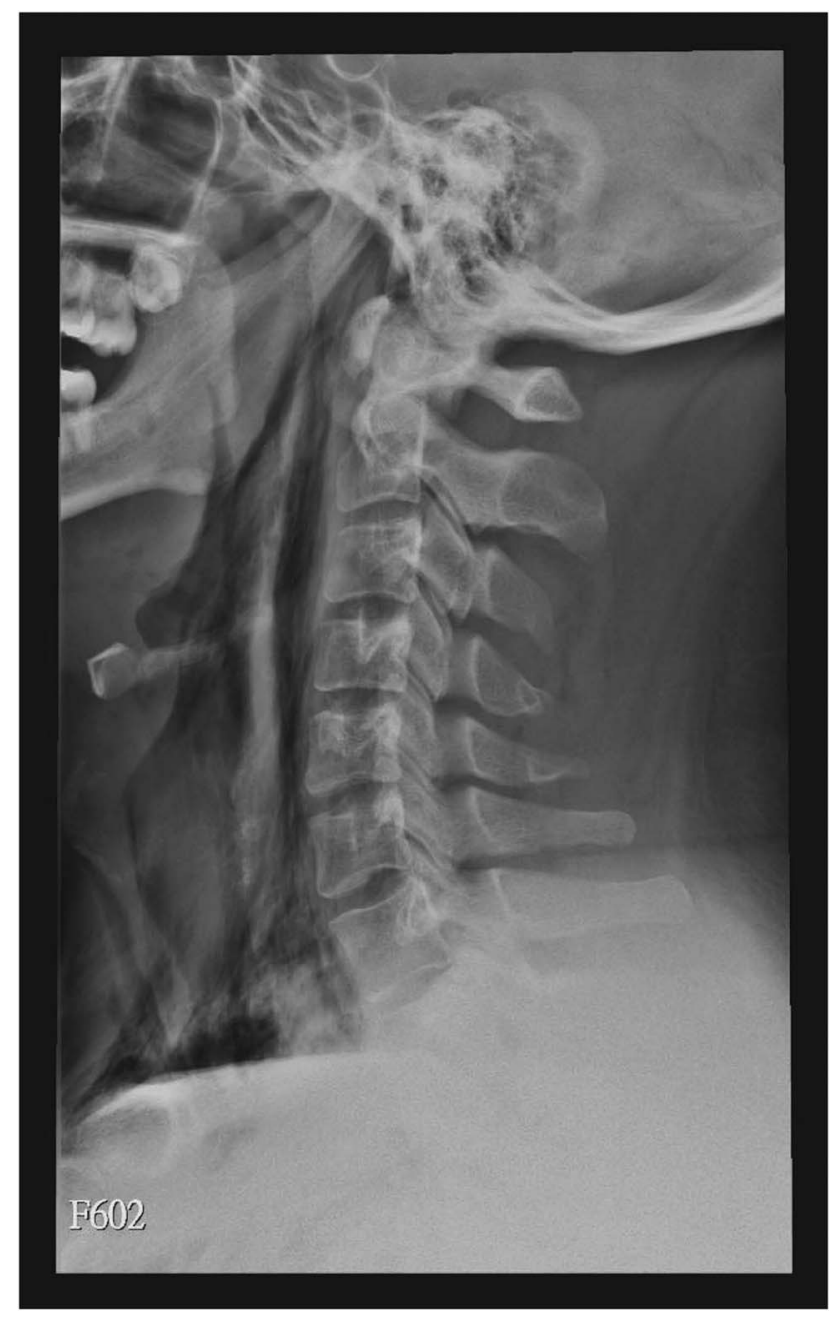

Figure 1. C-spine radiographs (left lateral view) show prominent subcutaneous and prevertebral emphysema from the base of the skull to the mediastinum.

treatment decisions like the need for urgent tracheostomy or fasciotomy.

Competing interests: None declared.

Keywords: Subcutaneous emphysema, dental extraction

From the *Department of Otorhinolaryngology, Head and Neck Surgery Department, Kaohsiung Veterans General Hospital, Kaohsiung, Taiwan.

Correspondence to: Dr. Ming-Yee Lin, Kaohsiung Veterans General Hospital, No. 386, Dazhong 1st Rd., Zuoying Dist., Kaohsiung City 813, Taiwan (R.O.C.); Email: mingyeelin@gmail.com 


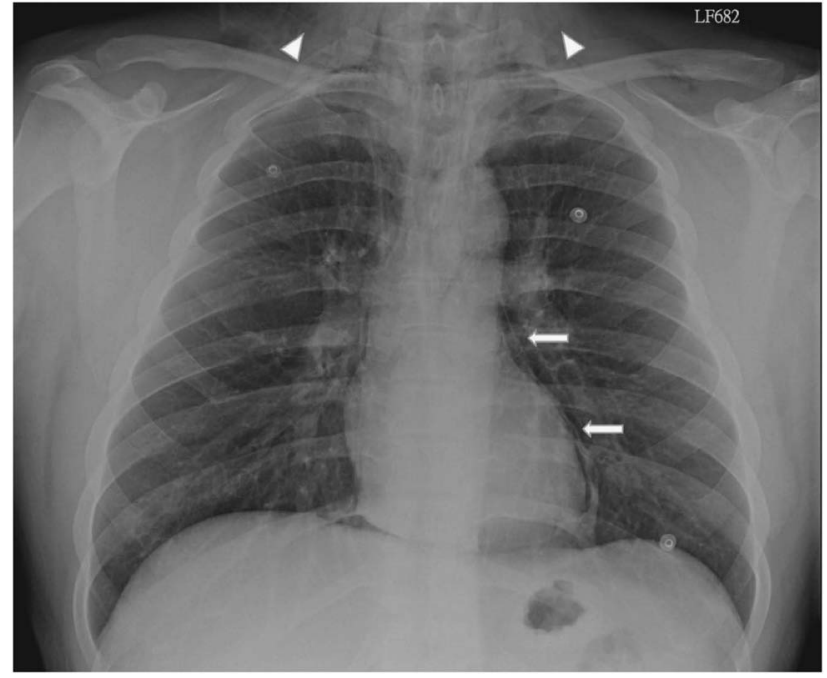

Figure 2. Posteroanterior chest radiograph shows subcutaneous emphysema (arrow heads) and pneumomediastinum (arrows).

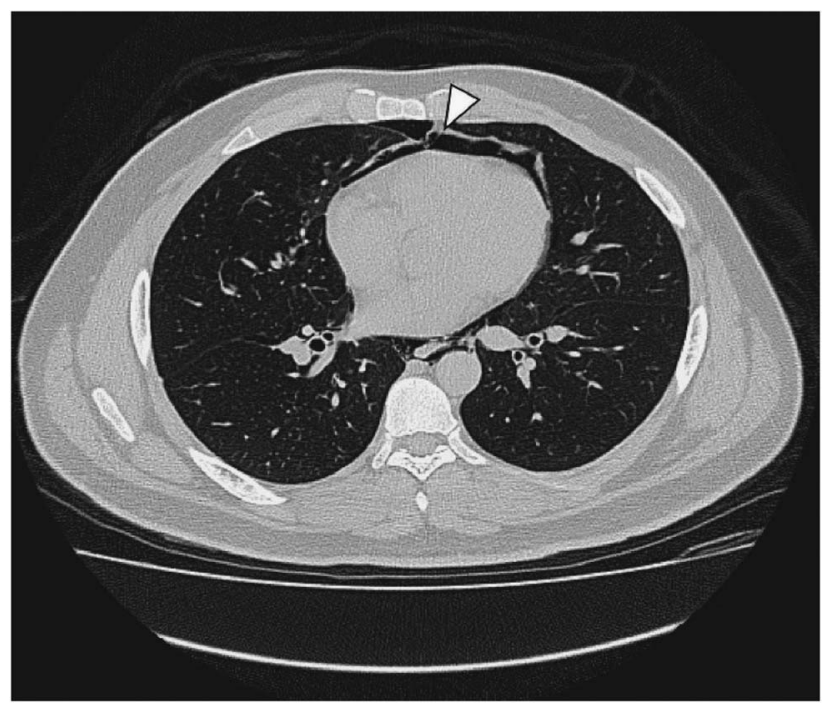

Figure 3. Non-contrast chest computed tomography shows pneumopericardium (arrowhead).

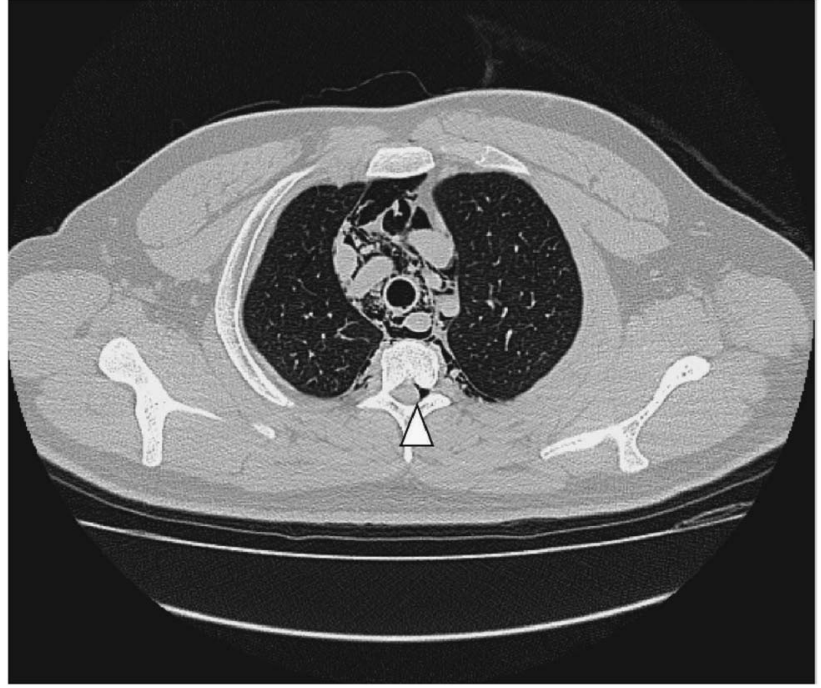

Figure 4. Non-contrast chest computed tomography shows gas collection at thoracic extradural space: pneumorrhachis (arrowhead).

\section{REFERENCES}

1. Goodnight JW, Sercarz JA, Wang MB. Cervical and mediastinal emphysema secondary to third molar extraction. Head Neck 1994;16:287-90; doi:10.1002/hed. 2880160314.

2. McKenzie WS, Rosenberg M. Iatrogenic subcutaneous emphysema of dental and surgical origin: a literature review. 7 Oral Maxillofac Surg 2009;67:1265. doi:10.1016/j. joms.2008.12.050.

3. Arai I, Aoki T, Yamazaki H, et al. Pneumomediastinum and subcutaneous emphysema after dental extraction detected incidentally by regular medical checkup: a case report. Oral Surg Oral Med Oral Pathol Oral Radiol Endod 2009;107:e33. doi:10.1016/j.tripleo.2008.12.019.

4. Ehmann MR, Paziana K, Stolbach AI. Case series: pneumorrhachis secondary to spontaneous pneumomediastinum. 7 Emerg Med 2016;50(2):e43-5; doi:10.1016/j. jemermed.2015.09.047. 